\title{
Calculation of Optimal Number of Tapes in Stack of Superconducting Tapes Subjected to Tilted Magnetising Field
}

\author{
L. Tomkow*, A. Smara, V. Climente-Alarcon and B.A. Glowacki \\ Applied Superconductivity and Cryoscience Group, \\ Department of Materials Science and Metallurgy, University of Cambridge, \\ 27 Charles Babbage Road, Cambridge CB3 OFS, United Kingdom
}

Doi: 10.12693/APhysPolA.138.737

*e-mail: lukasz.tomkow@cern.ch

\begin{abstract}
Stacks of superconducting tapes can be used as trapped-flux magnets and applied in a fully superconducting motor. In such application, they can be subjected to an angled magnetising field. In this work, the results of the numerical analysis of the stacks with different sizes, magnetised by angled magnetic fields, are presented. The numerical model applied bases on $H$-formulation. The optimum number of tapes in which the magnetisation per tape is the highest is sought. The dependence is obtained and presented for $40 \mathrm{~mm}$ wide tapes, similar in size to the ones applied in a future motor. It was shown that the optimal number of tapes increases with the strength of the magnetising field and decreases with its deviation from the direction normal to the stack.
\end{abstract}

topics: superconductivity, superconducting tapes, magnetic fields, magnetic flux trapping

\section{Introduction}

Stacked superconducting tapes can trap magnetic flux as high as $17.7 \mathrm{~T}[1]$. Such tapes are cheap, simple and stable both thermally and mechanically [2]. Trapped-flux magnets made of superconducting tapes will be used in a rotor of a fully superconducting synchronous airliner motor demonstrator [3], constructed under the ASuMED project. The application of superconductors in an aircraft motor is expected to lead to an increase of mass power density.

The stacks in the motor used for the ASuMED project will have complex shapes due to the need to decrease demagnetisation [4-6] and they will be affected by angled fields. In this paper, we investigate the effect of the number of tapes on magnetisation per single tape. The optimum number of tapes that allows for the maximum magnetisation is determined for a range of angles and magnetising fluxes with different strengths. The existence of such an optimum is hinted by previous experimental and numerical investigations [7].

\section{Methods}

A numerical model is developed in Comsol Multiphysics and uses 2D geometry presented in Fig. 1. The regions of the model are a stack, magnetising coils and an environment (modelled as air). The coils are moved around the stack by a certain angle $\alpha$. In the model description, we use $H$-formulation where stacks are modelled as anistotropic, homogeneous blocks $[8,9]$. The problem comes down to solve:

$$
\begin{aligned}
& \frac{\partial H_{x}}{\partial t}+\frac{\partial H_{y}}{\partial t}+\frac{\partial\left(E_{z}\left(J_{z}\right)\right)}{\partial x}-\frac{\partial\left(E_{z}\left(J_{z}\right)\right)}{\partial y}=0, \\
& E_{z}=\left\{\begin{array}{cc}
E_{0} \frac{J_{z}}{\left|J_{z}\right|}\left(\frac{\left|J_{z}\right|-J_{c}}{J_{c}}\right)^{n} & \text { when }\left|J_{z}\right| \geq J_{c}, \\
0 & \text { when }\left|J_{z}\right|<J_{c},
\end{array}\right. \\
& J_{z}=\frac{\partial H_{x}}{\partial y}-\frac{\partial H_{y}}{\partial x},
\end{aligned}
$$

where $H$ is the local strength of the magnetic field, $E$ is the electric field, $J$ is the current density and $t$ is the time. The $z$-component of the electric field is denoted as $E_{z}$ (out of model plane), $J_{c}$ is the critical current, $n$ is taken as 31 and $E_{0}$ is assumed as $100 \mu \mathrm{V} \mathrm{m}^{-1}$ [10]. Calculation of the critical current density is performed with the use of (4) and (5) from [11]. In turn, $J_{c 0}$ and $B_{0}$ are found using experimental data from [12]. Coefficients $\alpha, \beta$ and $\gamma$ are $1,0.67$ and 2.77 , respectively. In order to consider anisotropy of the tape, a directional parameter $\epsilon$ is introduced, hence

$$
\begin{aligned}
& J_{c}=J_{c 0}\left[1+\epsilon\left(\frac{B}{B_{0}}\right)^{\alpha}\right]^{-\beta}, \\
& \epsilon=\sqrt{\gamma^{-2}\left(\frac{B_{x}}{B}\right)^{2}+\left(\frac{B_{y}}{B}\right)^{2}},
\end{aligned}
$$




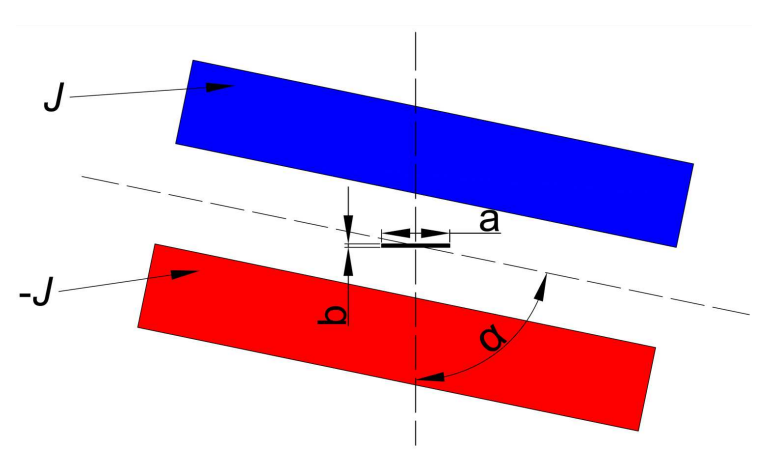

Fig. 1. Geometry of the model.

where $B$ is the local strength of magnetic induction. The analysed angles' range is between $0^{\circ}$ and $90^{\circ}$ and the number of tapes in the stack is between 1 and 25. The considered applied magnetic inductions $B_{\text {ap }}$ at the centre of the electromagnet (without the stack) equal 3.4, 1.7 and $0.85 \mathrm{~T}$.

\section{Results and discussion}

Figure 2 shows the magnetic flux trapped per single tape as the function of angle $\alpha$ of magnetising flux and its strength. Trapped flux generally decreases with $\alpha$ and only in some cases it remains constant within a certain range. This corresponds to the situation in which the stack remains fully magnetised despite the changing angle. In fact, when the number of tapes is larger, the trapped flux decreases faster with the increase of the angle. This is due to the fact that a part of the volume of the stack is not explored, if the magnetising flux is smaller than the penetration flux.

With the sufficiently high magnetising flux allowing full magnetisation, the trapped flux per tape increases with the tapes' number. This is probably related to the fact that although the tapes are placed internally, they are partially shielded and experience lower magnetic flux. Therefore, their critical current density is higher, allowing to trap a stronger flux. However in reality, this effect can be mitigated by the heat generation and the increase of temperature in the centre of the stack.

Figure 3 shows the estimated optimal number of tapes in the stack which allows to obtain the maximum magnetisation per tape. Importantly, the number of tapes decreases with the increase of $\alpha$ and increases with the increase of $B_{\text {ap }}$. In all $B_{\text {ap }}$ cases, the optimum number of tapes obtained with $\alpha=90^{\circ}$ is 1 . With such a value of the angle of the magnetising field, almost no flux is trapped, though. Our results also suggest that there exists the maximum number of tapes for which a further increase of $B_{\text {ap }}$ will not cause a further improvement of magnetisation per tape.

The maximum magnetisation per tape does not necessarily mean that the total trapped magnetic flux is the highest. In many cases, the entirely trapped flux will increase with the number of tapes.
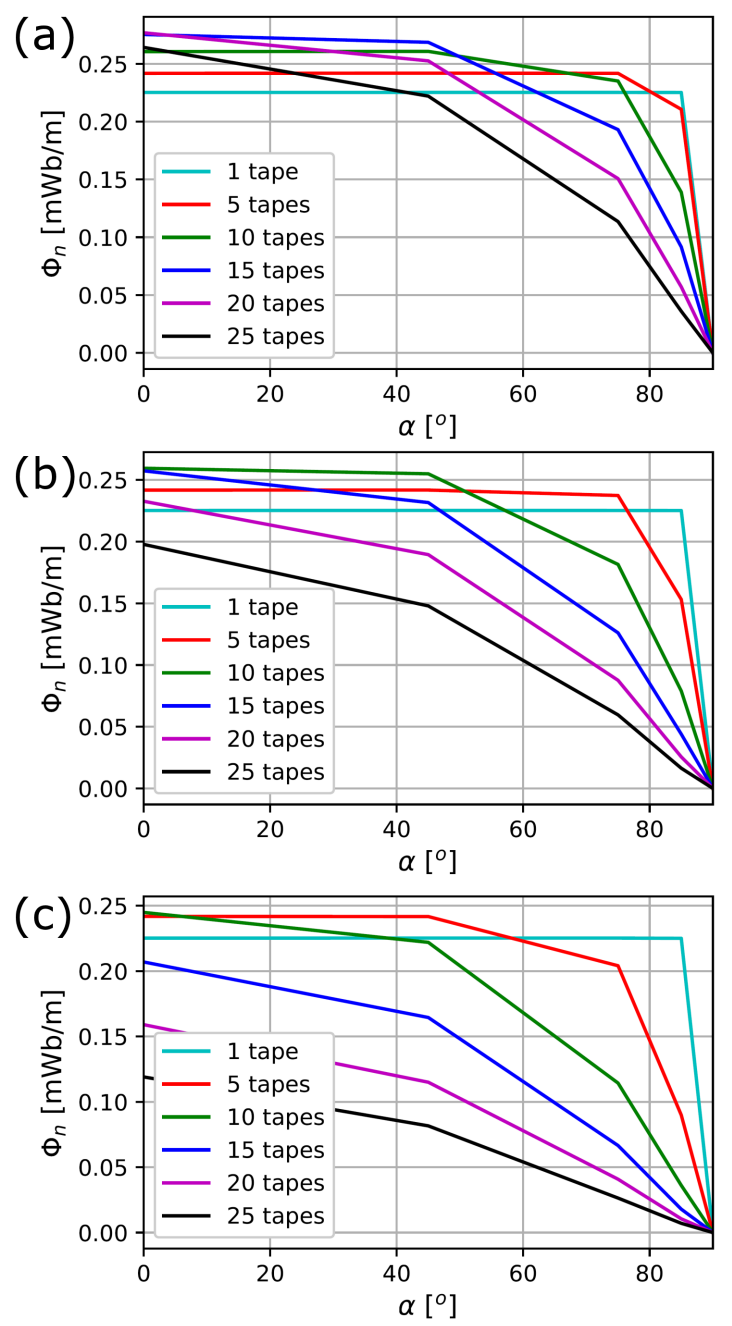

Fig. 2. Magnetic flux trapped per unit length of the stack for a single tape and for different values of $B_{\text {ap }}$ : (a) $3.4 \mathrm{~T}$, (b) $1.7 \mathrm{~T}$, (c) $0.85 \mathrm{~T}$.

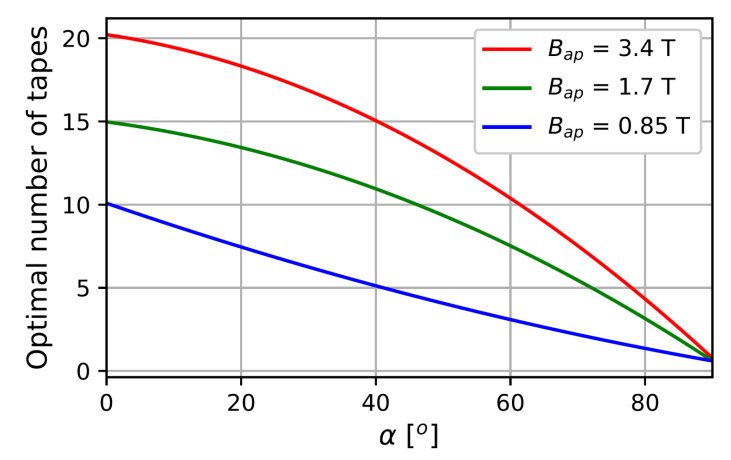

Fig. 3. Estimated optimal number of tapes with different $B_{\text {ap }}$.

Optimal magnetisation per tape, however, gives the highest ratio between the weight of the stack and the trapped field, the value crucial for aviation, marine and wind turbine applications. It marks the maximum number of tapes when the magnetisation is full for a given field. 


\section{Conclusions}

The obtained numerical results confirm the existence of an optimal size of the stack, which for the given combination of the angle and strength of the magnetising flux — yields the maximum magnetisation per tape. This value increases with the strength of the magnetising flux and decreases with its angle. When the optimal number of tapes is applied, the magnetisation is full and neither the magnetising field, nor the volume of the stack is wasted. The presented results are important in the context of the application of the stacks in the transport and energy industry as they allow for the minimisation of weight/flux ratio of the trapped-field magnets.

\section{Acknowledgments}

This research was partially supported by the European Union's Horizon 2020 Research and Innovation programme under grant agreement No. 7231119 (ASuMED "Advanced Superconducting Motor Experimental Demonstrator") and by EPSRC grant No. EP/P000738/1 entitled "Development of superconducting composite permanent magnets for synchronous motors: an enabling technology for future electric aircraft".

\section{References}

[1] A. Patel, A. Baskys, T. Mitchell-Williams, A. McCaul, W. Coniglio, J. Hänisch, M. Lao, B.A. Glowacki, Supercond. Sci. Technol. 31, 09LT01 (2018).

[2] A. Baskys, A. Patel, S.C. Hopkins, V. Kalitka, A. Molodyk, B.A. Glowacki, IEEE Trans. Appl. Supercond. 25, 1 (2015).
[3] V. Climente-Alarcon, A. Patel, A. Baskys, B.A. Glowacki, IOP Conf. Ser. Mater. Sci. Eng. 502, 012182 (2019).

[4] A. Patel, V. Climente-Alarcon, A. Baskys, B.A. Glowacki, T. Reis, in: Proc. IEEE ESARS-ITEC, Nottingham (UK), IEEE, 2018, p. 18403474.

[5] A. Baskys, A. Patel, B.A. Glowacki, Supercond. Sci. Technol. 31, 065011 (2018).

[6] A. Baskys, A. Patel, V. Climente-Alarcon, B.A. Glowacki, J. Supercond. Novel Magn. 32, 3071 (2019).

[7] L. Tomkow, A. Smara, V. ClimenteAlarcon, B.A. Glowacki, J. Supercond. Novel Magn. 33, 1299 (2020).

[8] R. Pecher, M. McCulloch, S. Chapman, L. Prigozhin, "3D-Modelling of Bulk Type-II Superconductors Using Unconstrained $H$-formulation", in: Proc. EUCAS 2003, 2003.

[9] V.M.R. Zermeno, A.B. Abrahamsen, N. Mijatovic, B.B. Jensen, M.P. Sørensen, J. Appl. Phys. 114, 173901 (2013).

[10] J. Kvitkovic, S. Patel, M. Zhang, Z. Zhang, J. Peetz, A. Marney, S. Pamidi,IEEE Trans. Appl. Supercond. 28, 1 (2018).

[11] X. Zhang, Z. Zhong, J. Geng, B. Shen, J. Ma, C. Li, H. Zhang, Q. Dong, T.A. Coombs, J. Supercond. Novel Magn. 31, 3847 (2018).

[12] M. Zhang, J.H. Kim, S. Pamidi, M. Chudy, W. Yuan, T.A. Coombs, J. Appl. Phys. 111, 083902 (2012). 